\title{
Actitudes hacia el plagio en estudiantes de Administración de Empresas de dos universidades privadas en Arequipa
}

\section{Attitudes towards Plagiarism in Business Administration Students from Two Private Universities in Arequipa}

\author{
Teresa Ramos Quispe* (i) \\ Universidad Continental, Arequipa, Perú \\ ORCID: https://orcid.org/0000-0003-4607-4745 \\ Edgar Froilan Damián Núñez \\ Universidad Nacional Mayor de San Marcos, Lima, Perú \\ ORCID: https://orcid.org/0000-0001-7499-8449 \\ Miguel Gerardo Inga Arias \\ Universidad Nacional Mayor de San Marcos, Lima, Perú \\ ORCID: https://orcid.org/0000-0002-1588-0181 \\ Dennis Arias Chávez \\ Universidad Continental, Arequipa, Perú \\ ORCID: https://orcid.org/0000-0003-1500-8366 \\ María Jesús Caurcel Cara \\ Universidad de Granada, Granada, España \\ ORCID: https://orcid.org/0000-0002-2169-9100
}

Recibido 15-07-18 Revisado 02-08-18 Aprobado 17-11-18 En línea 06-12-18

Correspondencia:

Email: tramos@continental.edu.pe
Citar como: 


\section{Resumen}

El presente estudio tiene por objetivo identificar, describir y explicar las actitudes hacia el plagio de los estudiantes de la carrera de Administración de Empresas de dos universidades privadas en Arequipa. Se emplea una metodología cualitativa y la técnica de análisis de contenido. Los datos se han obtenido a partir de la información proveniente de la aplicación de una guía de entrevista individual y una guía de grupo focal. Se han realizaron 20 entrevistas individuales y 2 grupos focales a un total de 20 estudiantes ingresantes (10 mujeres y 10 varones). Las categorías surgidas del discurso expresado por los participantes son: (a) conocimiento del fenómeno; (b) actitud permisiva hacia el plagio; (c) el plagio como facilitador social; (d) consecuencias; y (e) actitud preventiva. Los resultados muestran que, para los entrevistados, el plagio es definido como el acto de "copiar algo que no es de uno", "hacer trampa" o "robar". Asimismo, concluyeron que plagiar es una práctica común e incluso institucionalizada que empieza en el colegio y se perfecciona en la universidad. Obtener buenas notas es la razón por la que no existen acusaciones ni discrepancia entre los estudiantes que cometen plagio y los que no lo hacen, ya que para ellos plagiar no es un delito. Finalmente, el desconocimiento de que se está cometiendo plagio, el facilismo y el temor a desaprobar son algunas de las causas de este acto. Entre las propuestas que los entrevistados mencionan para solucionar este problema se encuentra que las universidades divulguen y apliquen sus reglamentos disciplinarios.

Palabras clave: Plagio académico; prácticas de escritura; educación superior; trabajos académicos.

\section{Summary}

The present study is aimed to identify, describe and explain attitudes toward plagiarism of the students of Business Administration from two private universities in Arequipa. A qualitative methodology was used and the technique was the analysis of content. The data were obtained from information from the application of an individual interview guide and a guide to focus group. Twenty individual interviews and two focus groups were conducted, totalling 20 newly-admitted students (10 women and 10 men). The categories arising from the answers expressed by the participants were: (a) knowledge of the phenomenon, (b) permissive attitude toward plagiarism, (c) plagiarism as a social facilitator, (d) consequences and (e) preventive attitude. The results show that, for those interviewed, plagiarism is defined as the act of "copying something that does not belong to one", "cheat" or "theft". They also concluded that plagiarizing is a common and even institutionalized practice that begins at school and is perfected in the University. Getting good grades is the reason why there are no accusations or discrepancy between students who commit plagiarism and those who do not, since they maintain that plagiarism is not a crime. Finally, the ignorance that you are committing plagiarism, the superficiality and fear to fail a course are some of the causes of this cheating action. Among the proposals that the interviewees mentioned to solve this problem is for universities to disclose and implement its disciplinary regulations.

Keywords: Academic Plagiarism; Writing Practices; Higher Education; Academic Works.

\section{Introducción}

Las actitudes son predisposiciones adquiridas para actuar selectivamente y conducirse de una forma determinada en el proceso de interacción social (Vander, 1997). Se las define, también, como un estado mental y neutral de disposición que se organiza mediante la experiencia, la cual ejerce una influencia directa en la respuesta de la persona a todos los objetos o situaciones con los que se relaciona (Allport, 1977). En los últimos años, se han perpetuado prácticas deshonestas en la labor académica y científica. Tomar las ideas de otros sin citar su procedencia parece ser un hábito común entre estudiantes tanto de educación secundaria como de nivel superior. Y aunque la preocupación por las implicancias éticas y legales que este acto conlleva parece ir en aumento, muy pocos son conscientes de su gravedad. Son muchas las creencias o sentimientos que un 
estudiante tiene de las consecuencias de cometer un acto deshonesto, sobre todo cuando este conlleva una sanción social.

El avance de la informática ha permitido que millones de usuarios en el mundo puedan compartir información en tiempo real. A esto se suma, la facilidad con que una persona puede hacer visibles sus publicaciones, aunque con el riesgo de que sus aportes sean tomados por otros sin darle el crédito respectivo. Este es el mayor riesgo que se corre al navegar en internet; por ello, universidades y otros centros de enseñanza superior vienen implementando sistemas de control que ayuden a disminuir los casos de plagio entre estudiantes y profesorado. En 2016, la empresa Turnitin ('Entrégalo', en inglés) publicó un estudio sobre el índice global de plagio en la educación secundaria y superior. Para el caso de América Latina, de un total de 196.691 trabajos analizados, el porcentaje de contenido no original hallado fue de $12 \%$, cantidad solo superada por Asia Oriental, región que presenta un $14 \%$. Estos porcentajes muestran que el plagio no es un problema regional o local, sino global.

Diversos estudios han evidenciado la gravedad de esta práctica. En 2011, Molina, Velásquez, Ríos, Calfucoy y Cociña determinaron que más del 50\% de estudiantes chilenos de educación secundaria y el $40 \%$ de educación superior admitieron haber tomado información de internet sin citar la fuente. Otros resultados que confirman este problema son los de Sureda et al., quienes en 2009 realizaron un estudio en una universidad mexicana con estudiantes de 15 años en adelante, el cual arrojó que más del $44 \%$ de estudiantes habían cometido "ciberplagio" (información tomada de internet sin citar), en tanto que el 30\% admitió haber "copiado y pegado" información de internet en sus trabajos académicos. Ambos estudios coinciden en afirmar que entre las causas que llevan a un estudiante a cometer plagio están "el ahorro de tiempo", "confianza personal", "la facilidad que ofrece el internet para hacerlo", "la costumbre de hacer las cosas a último momento", "obtener mejores calificaciones", "imitación al hacer lo que otros hacen sin tener repercusiones", "carencia de políticas e instrumentos institucionales para la detección y tratamiento de este acto deshonesto", entre otras (Ercegovac \& Richardson, 2004; Lai \& Weeks, 2009; Ma, et al.; 2008; Pupovac, et al., 2008; Batane, 2010). No obstante, es necesario precisar que, entre todas estas causas, sobresale la relacionada con la pereza, tal y como lo menciona un estudio hecho por Egaña (2012), en el que tanto docentes como estudiantes consideran como causa del plagio la ley del mínimo esfuerzo en la que se prefiere "copiar y pegar" o resumir y parafrasear inadecuadamente en lugar de dedicarle el tiempo suficiente a leer y profundizar en diferentes fuentes para realizar sus deberes e investigaciones (Soto, 2012; Molina et al., 2011). A estas causas habría que añadir la labor del docente como corresponsable de este fenómeno al asignar a los alumnos tareas sobre temas generales de su asignatura y a las instituciones, que son permisivas con este acto, lo que lleva a que la comunidad lo considere como una actividad "normal" e incluso natural.

Los estudios antes mencionados se complementan con aquellos relacionados con la escritura deshonesta (Ochoa \& Cueva, 2014; Espinosa, Castellarin, \& Biagioni, 2013), los que abordan el tema de la percepción sobre el plagio académico en estudiantes universitarios (Castro, Yoplac-López, Carpio-Tello, Sihuay-Torres \& Cósar-Quiroz, 2017; Cebrián-Robles, RaposoRivas, Cebrián de la Serna \& Sarmiento-Campos, 2018), también los estudios sobre las atribuciones causales en el plagio académico por parte de los estudiantes universitarios (RebolloQuintela, Espiñeira-Bellón \& Muñoz-Cantero, 2017) y estudios cuyo interés se centra en diseñar instrumentos para medir el plagio académico entre estudiantes universitarios (Cayuela, Tauste, Seguí, Esteve \& Ronda, 2015).

El plagio, considerado como acto deshonesto, ha sido denunciado tanto por instituciones académicas como por no académicas. Dada la gravedad y repercusión del hecho, sus implicaciones en la actualidad van más allá de sanciones reglamentarias, y su alcance llega a lo legal y penal. Al respecto, el Código Penal Peruano, en su artículo 219, dice textualmente sobre el plagio: 
Será reprimido con pena privativa de libertad no menor de cuatro ni mayor de ocho años y noventa a ciento ochenta días multa, el que, con respecto a una obra, la difunda como propia, en todo o en parte, copiándola o reproduciéndola textualmente, o tratando de disimular la copia mediante ciertas alteraciones, atribuyéndose, o atribuyendo a otro, la autoría o titularidad ajena (Ley 28289, 2004).

El ámbito académico peruano no se ha mantenido ajeno a este problema, y han sido las universidades quienes se han pronunciado al respecto. Ahora bien, esta reacción es reciente y se ha producido a raíz de una serie de casos que, en su mayoría, se han dado en el ámbito político. No son pocas las instituciones que han quitado grados y títulos a determinados personajes tras haber descubierto, gracias a la prensa, certezas de plagio en tesis y artículos científicos (el caso más sonado fue la destitución de la jefa de la SUNEDU ${ }^{1}$ por un caso de plagio). Esta preocupación se manifiesta por el aumento de la demanda de software para prevenir el plagio por parte de entidades de educación superior peruanas. La transición de la sociedad analógica a una digital y la cantidad de información a la que un estudiante puede tener acceso parecen indicar que el problema del plagio irá en aumento y en muchas más modalidades.

A este problema se suma la necesidad por la especialización, razón que viene obligando a que aumente la demanda por estudiar maestrías y doctorados y obtener a cualquier costo los grados. Al respecto la SUNEDU, en un comunicado publicado en abril del 2018, indica que es responsabilidad de las universidades implementar políticas y mecanismos de protección a la propiedad intelectual señaladas en su propia normativa, tales como Estatutos, Reglamento de Grados y Títulos y Código de Ética para la investigación. Asimismo, dicha Superintendencia recomienda a las universidades resolver denuncias y reclamos sobre supuestos plagios en las tesis con el fin de evaluar el grado de certeza y responsabilidad de los involucrados.

Si bien existe un avance institucional por combatir este mal, aún queda por hacerlo desde el ámbito de los colegios, ya que estos representan la base de la educación formativa y su presencia es notoria entre niños y jóvenes desde la educación básica a la universidad (Díaz, 2015). El estudiante que ingresa a la universidad traslada su experiencia colegial en las labores propias de la universidad. En él no existe aún conciencia de que plagiar en un examen o tomar las notas de sus compañeros sin dar el crédito correspondiente represente una falta grave, como también desconoce cuáles son las formas en que se presenta el plagio, cuál es su alcance ético-moral y cómo debe prevenirlo. Estos fenómenos sientan las bases de una actitud permisiva con el plagio, actitud que se verá reflejada en los años superiores e incluso fuera de la universidad.

\section{Método}

La presente investigación se inscribe dentro del enfoque cualitativo. Los datos se obtuvieron a partir de la información proveniente de la aplicación de una guía de entrevista individual y una guía de grupo focal. Se realizaron 20 entrevistas individuales y dos grupos focales a un total de 20 estudiantes ingresantes (10 mujeres y 10 varones) a la carrera de Administración de Empresas de dos universidades privadas en Arequipa. Estos fueron seleccionados de acuerdo a criterios no probabilísticos, utilizando un muestreo intencional. Las edades de los estudiantes oscilan entre los 17 y 22 años, de diferentes niveles socioeconómicos.

En lo que refiere a la validez de las técnicas utilizadas en el presente estudio, la entrevista cualitativa tiene por objetivo entender el significado de los temas centrales en el mundo vivido de los sujetos (Kvale, 2011). En este proceso, es el entrevistador el responsable de registrar e interpretar los significados que los participantes construyen en su discurso, además de evaluar la forma como lo dicen. Para ello, se hace necesario que el entrevistador conozca el tema tratado, así como los principios fundamentales del proceso: observar e interpretar. Para fines prácticos, la

\footnotetext{
${ }^{1}$ Superintendencia Nacional de Educación Superior Universitaria, organismo que supervisa y controla la marcha de las universidades del Perú.
} 
información recolectada para el estudio se obtuvo en los entornos reales de los participantes, como es su centro de estudios, lo que permite contextualizar mejor el fenómeno.

\section{Procedimiento.}

La recolección de los datos se realizó durante los meses de agosto y setiembre de 2018. Entre las primeras actividades realizadas por el equipo de investigación estuvo el elaborar las guías de entrevista. Para cumplir con los objetivos de esta primera etapa se contó con cuatro colaboradoras adicionales (dos por cada universidad) que tuvieron las funciones de entrevistador y observador, además de ser las encargadas de gestionar los ambientes destinados para el proceso investigativo. Ambas fueron capacitadas por un miembro del equipo investigador en materia de manejo de los equipos de grabación y apoyo en el proceso de entrevista. Para los grupos focales, se solicitó a ambas universidades un ambiente silencioso con buena iluminación y mobiliario adecuado.

La realización de la entrevista personal formó parte de la segunda etapa del estudio y duró doce días. Para ello, se utilizaron las mismas aulas facilitadas por la universidad, previa coordinación con el responsable asignado por la institución. Se cuidó que cada entrevista se realizara en las mismas condiciones, con los mismos equipos y respetando los protocolos elaborados, con el fin de que las respuestas sean lo más sinceras posibles.

La realización del grupo focal formó parte de la segunda etapa, en lo cual se respetaron los protocolos establecidos para la entrevista individual en materia de ambientación y manejo de equipos. Cada grupo focal tuvo una duración de 30 minutos. Una vez que se obtuvo la información de los participantes, se procedió a transcribirla en formato Word. Para su edición se respetaron las construcciones sintácticas y léxicas propias del discurso oral. Finalmente, se realizó el procesamiento de la información.

Es importante señalar que el procesamiento de datos cualitativos se basa en procesos y operaciones lógicas analíticas inductivas (Gibbs, 2012). Por ello, la información se analizó a través de la metodología del Análisis de contenido, el cual es una técnica de investigación "destinada a formular, a partir de ciertos datos, inferencias reproducibles y válidas que puedan aplicarse a su contexto" (Krippendorf, 2002, p. 28). Para dar fiabilidad al análisis de la información recolectada, cada investigador analizó cada una de las entrevistas y estableció categorías que fueron surgiendo de los datos. Las categorías se formaron del discurso expresado por los participantes a través de la comparación constante de las unidades de análisis. Para este fin se asignaron códigos, y se procedió a establecer categorías primarias o descriptivas y categorías analíticas. Las primeras emergieron de las verbalizaciones de los participantes; en tanto que las segundas, de procesar las primeras. Este segundo nivel de categorización se basó en la comparación constante de las categorías primarias, de tal forma que algunas de ellas se integraron en conceptos más abstractos y explicativos. Luego, se relacionaron entre sí las categorías finales para obtener patrones temáticos (categorías principales que tienen entre sí relaciones de causaefecto, implicancia, coocurrencia, etc.).

Una vez establecidas las categorías, se procedió a la codificación del material. La decisión final se tomó a partir del acuerdo de los investigadores, tomando en cuenta los puntos coincidentes, por ejemplo, si se otorgaba la misma categoría a la porción de contenido analizada. De este análisis surgieron las siguientes categorías y subcategorías: conocimiento del fenómeno (definición y ejemplos), actitud permisiva hacia el plagio (en relación con determinadas actividades académicas), el plagio como facilitador social (en relación a la posibilidad de obtener beneficios al plagiar), consecuencias (relacionadas con las sanciones que debe recibir quien plagia), actitud preventiva (causas y consejos para evitar el plagio).

La calidad de una investigación depende del rigor con el que esta se realice, ya que los procedimientos que se elijan para llevarla a cabo condicionarán su credibilidad. Ahora bien, los criterios que se manejan en las investigaciones cuantitativas difieren de los estudios de enfoque 
cualitativo. Entre los criterios que debe seguir todo estudio cualitativo se encuentran la credibilidad y la confirmabilidad (Guba, 1981), los cuales se logran garantizando que existan evidencias reales de los fenómenos estudiados, así como el acceso a ellas. En este sentido, el presente estudio procedió a registrar en audio y video cada una de las entrevistas realizadas, así como a transcribir lo dicho por los participantes respetando las características propias del discurso oral.

Asimismo, se utilizó la triangulación de técnicas de recolección de datos tal como es la entrevista cualitativa individual y el grupo focal, lo cual puede contrastarse en la recuperación de unidades de análisis donde hay ejemplos de discursos individuales y del grupo focal. Tal y como se mencionó líneas arriba, se procedió también a triangular el análisis realizado por los investigadores y colaboradores con el fin de tomar decisiones que uniformicen sus resultados. Es importante resaltar que durante estas sesiones surgieron diferencias, sobre todo de percepción en el proceso de la elaboración de las categorías primarias, pues estas exigen que se filtre toda la información de una manera puntillosa.

Las guías de entrevista se aplicaron sin distinciones ni en forma ni en proceso a cada uno de los participantes, lo cual ayudó a lograr la dependencia del estudio. Asimismo, se supervisó de manera sistemática cada una de las etapas del estudio, tanto en la organización como en el desempeño de los colaboradores.

La investigación cualitativa acepta que el objeto de la investigación es un sujeto interactivo, motivado e intencional, que asume una posición frente a las tareas que enfrenta (Parra y Briceño, 2013). Es por ello que la presente investigación garantiza la participación voluntaria de los participantes y el cuidado de su dignidad y bienestar durante el proceso, y para ello se obtuvo el consentimiento informado escrito en el que se resaltan la confidencialidad de los datos y el anonimato de los participantes.

\section{Resultados}

\section{Definición del fenómeno.}

El plagio se ha definido tradicionalmente como el acto de tomar las ideas de otros y hacerlas pasar por nuestras. Esta definición resalta el hecho de que plagiar implica engaño y usurpación de una obra ajena. En la universidad, este acto se manifiesta en actividades como evaluaciones, exposición de trabajos y en la redacción de monografías y tesis. Estas definiciones coinciden con lo manifestado por los entrevistados, quienes definieron el plagio como un acto de "copiar algo que no es de uno" y "hacer trampa" o "robar", estas últimas usadas como expresiones agravantes del hecho. Cabe señalar que estas categorías se encontraron tanto en las entrevistas individuales como en los dos grupos focales.

Es una copia, copiar algo de otro. Como en un examen, le miras la prueba a tu compañero para copiarlo. Copiar cosas de las tesis y de libros. Copiar tal y cual sin cambiar nada (S1).

En pocas palabras es hacer trampa, no ser sincero con uno mismo en un examen (S9).

Mirar el examen de un compañero, tomar notas o miras tus notas durante el examen (S12).

Es tener apoyo en un examen, fórmulas, notas escondidas para que el docente no los sepa, con el fin de que no jalemos. Plagiar es robar lo que hicieron otros (S10).

Copiar, hacer trampa, copiar en una tesis sin citar las fuentes. Cuando en un examen copias las respuestas (Grupo focal 1).

Ahora bien, cuando se les solicitó un ejemplo de plagio, la mayoría de los entrevistados brindaron como casos en los que se extrae información y se la altera con el fin de hacerla pasar como propia. Si bien se concibe el plagio como actividad deshonesta, su ejecución se encasilla en 
el ámbito del manejo de la información, dejando de lado otras situaciones mucho más complejas. Las respuestas refuerzan la idea de que plagiar es un acto de tomar lo que no es de uno sin reconocer al autor, en situaciones como

\author{
Sacar información de Internet sin citarla. \\ No poner comillas (S4). \\ Cuando saca información de un trabajo que no es de uno (S17). \\ Se cita literalmente y no se indica la fuente de información (S10) \\ Cuando se redacta usando o cambiando algunas ideas de una fuente escrita y no se \\ reconoce la fuente de la información (Grupo focal 2).
}

\title{
Actitud permisiva hacia el plagio.
}

Esta categoría se relaciona con determinadas actividades académicas que justifican la acción de plagiar. La tolerancia no es unidireccional, es decir, no se da el "yo tolero que otros plagien sin importarme que ellos sean tolerantes conmigo", sino "yo tolero que otros plagien porque yo también lo hago o lo puedo hacer". Existe una posición clara entre los estudiantes. Por un lado, no justifican este acto, ya que lo consideran "malo"; sin embargo, al preguntárseles si "plagiar es normal", los entrevistados aceptaron que es una práctica común e incluso institucionalizada que empieza en el colegio y que en la universidad llega a convertirse en una necesidad. Esta aparente "necesidad de plagiar" queda evidenciada en las siguientes respuestas:

Para los ingresantes es normal. He sido testigo, mis compañeros lo hacen porque les ayuda, los beneficia (S19).

No se justifica. Pero es normal entre los estudiantes que ingresan quizá por el temor de ser menos que los demás (S12).

Creo que no porque uno se esfuerza por hacer sus trabajos. Aunque muchos ven una salida fácil para aprobar. Existe temor entre los estudiantes por jalar (S9)

Al principio hay miedo por ser descubierto, pero más adelante agarran cancha $y$ aprenden mejor a plagiar (Grupo focal 1).

La permisividad es un factor macrosocial, esto es, se justifica porque es una condición tácita para pertenecer a un grupo: existe un acuerdo tácito entre los estudiantes de no delatar a sus compañeros. Esta permisibilidad no necesariamente lleva a la aceptación directa; una estrategia es la indiferencia, tal y como se puede observar en las siguientes respuestas:

Este acto es normal no solo en ingresantes sino también en todos (S14).

Me es indiferente, si veo plagiar a otros no es mi problema (S12).

No he visto plagiar a nadie. Y si lo veo me sería indiferente (S11)

Si he visto plagiar, pero me ha sido indiferente (S20)

He sido testigo, mis compañeros lo hacen porque les ayuda, los beneficia. Creo que lo hacen por desconocimiento. Me es indiferente (S3).

\section{Facilitador social.}

Esta categoría se relaciona con la posibilidad de obtener beneficios, ya sean sociales o grupales, al plagiar. Existen algunos puntos en los que los entrevistados coinciden, por ejemplo, en que no existen diferencias en el trato hacia aquellos que plagian; hasta cierto punto, el acto de copiar en los exámenes y en los trabajos no representa un problema, prueba de ello es que no se dan tratos diferenciados entre quienes plagian y quienes no. Por otra parte, la complicidad se alza como una conducta común entre el grupo. Todos apuntan a un objetivo: sacar buenas notas, es por ello que, al trabajar en equipo, se pasa por alto este y otros actos.

Los integrantes no actúan diferente porque lo ven normal, porque como todos lo hacen, es normal. Sí hay complicidad, porque sabemos que todos plagian (S12). 
No hay diferencias. Hay complicidad para cometer plagio, son testigos, pero no dicen nada, quizá porque todos tienen la intención de plagiar en algún momento. Esto se manifiesta sobre todo en los trabajos grupales (S16).

No actúan diferente, porque lo ven normal, porque como todos lo hacen es normal. Sí hay complicidad, porque sabemos que todos plagian. Podrías conseguir, pero no serían verdaderos los logros (S2).

A veces hay cólera cuando vemos a alguien plagiar porque se la "lleva fácil", pero cuando es solidario y ayuda a todos ya no hay enojo (Grupo focal 1).

De las respuestas podemos inferir que este acto "normal" conlleva implícito el concepto de "aceptación social" o "ser parte de" que se da como producto de las valoraciones sociales hechas por el grupo frente a diferentes situaciones y contextos experimentados en la universidad. Es importante resaltar que las actividades que más se relacionan con el acto de plagiar son la resolución de exámenes y el desarrollo de trabajos, ya sean individuales o grupales. El logro inmediato que se obtiene al copiar en un examen es tener buenas calificaciones; por tanto, cualquier acto que apunte a alcanzar este logro será permitido por el estudiante y el grupo.

Plagiar trae beneficios, por ejemplo, si sacas buenas notas tienes becas, así que eso lleva a la gente a querer plagiar (S18).

Los entrevistados coinciden en afirmar que los perjuicios se verán a largo plazo, esto es, en la vida profesional.

Plagiar no trae cosas buenas, te da mala reputación. Puedes obtener buenas notas, pero en la vida profesional tendría problemas (S9).

\section{Consecuencias.}

Esta categoría de relaciona con las sanciones que recibe quien plagia. A la pregunta de si el plagio era considerado un delito, las respuestas fueron unánimes: "plagiar no es un delito", al menos no en la universidad, ya que este acto es normal y necesario.

[Plagiar] No es un delito, pero si es un acto que está mal. Académicamente, no creo que sea un delito (S7).

El plagio no es un delito. Algunos plagian por necesidad (S8).

Plagiar no es un delito. Entre los estudiantes no es delito plagiar porque es común (Grupo focal 1).

Ahora bien, existen también posturas que sostienen que el plagio es un delito, o que no es, dependiendo de la situación. Para los entrevistados, los productos académicos desarrollados en los cursos no son considerados importantes, es por ello que plagiar durante su desarrollo no es tan grave. Surge aquí la tesis como producto con estándares altos en el que el plagio no se puede permitir.

Depende, plagiar es un delito cuando copias en un examen. Pero plagiar en una tesis sí es un delito grave (S10)

Plagiar es un delito solo para trabajos complejos como la tesis, es decir, si tu trabajo va a ser publicado no se debe plagiar (Grupo focal 2).

Ahora bien, cuando se les preguntó si plagiar perjudica, la gran mayoría sostuvo que sí, que la acción de plagiar es un problema que alcanza su máxima expresión en la vida académica. Esta respuesta lleva a suponer que si ha de haber una sanción para el plagiador, esta será una vez que el infractor esté fuera de la universidad. Esta respuesta se ve respaldada por los propios estudiantes cuando afirman que desconocen la existencia de un reglamento de la universidad en el que se precise el castigo para quien plagia. 
Plagiar perjudica más, ya que demuestra que una persona no sabe ni conoce de los temas (S3). El plagio perjudica, pero solo en ciertas cosas ya que podría conseguirte cosas en la vida (S19). Plagiar perjudica porque lo ve como un hábito y en su profesión lo seguirá haciendo (S2). No sé si la universidad tiene un reglamento. No conozco que haya un reglamento. No sé si hay un reglamento $(\mathrm{S} 18)$.

\section{Actitud preventiva.}

Esta categoría se relaciona con las causas y medidas propuestas por los entrevistados para evitar el plagio. El plagio es visto por los entrevistados como una forma de "atajo" para llegar al éxito académico y una demostración de astucia de quien lo comete. A esto habría que sumar la idea que tienen los estudiantes de que plagiar no es malo, siempre y cuando sea justificado, por ejemplo, cuando no se le entiende a un profesor o cuando los cursos son, en su opinión, complejos. Si se tuviera que hacer un balance entre los beneficios y perjuicios de plagiar, los entrevistados se inclinan por lo primero. Ahora bien, al preguntárseles a los entrevistados sobre cuáles serían las principales causas del plagio, estos propusieron tres causas puntuales: el desconocimiento de que se está cometiendo plagio, el facilismo y el temor a desaprobar:

Las causas son el desconocimiento, tanto porque no saben que plagiar es malo (S14).

El desconocimiento es la mayor causa (S2).

El facilismo, es más fácil plagiar que estudiar (S18).

Los alumnos saben que no se debe plagiar, pero por sacar buenas notas lo hacen (S20)

La causa principal es por no estudiar. Desconocer sobre lo malo que es plagiar, el factor es por no haber estudiado (S19).

La causa que lleva a cometer plagio es la presión de querer aprobar y la flojera, buscan la forma fácil de pasar los exámenes (Grupo focal 2).

En lo que respecta a las acciones para evitar el plagio, existen posiciones encontradas ya que, por un lado, se encuentran los estudiantes que por considerarlo un acto "normal" piensan que no debería ser castigado. Y por otro lado, están los que proponen un castigo, aunque este no conlleve expulsión o sanción, sino que la punición se manifieste en una nueva oportunidad para rendir el examen o presentar el trabajo. Estas respuestas refuerzan la complicidad que existe entre los estudiantes. Asimismo, las universidades tienen un reglamento en el que se precisan las sanciones para los estudiantes que cometan plagio. Si bien estos documentos son de consulta obligatoria por los estudiantes, todos los entrevistados afirmaron que jamás habían recibido dicho reglamento. Son precisamente estos documentos lo que los entrevistados afirman ayudarían a reducir la incidencia de este problema.

Si hay un documento que diga que plagiar trae castigo, pocos lo cometerían (S17). No sé si la universidad tenga un reglamento, no lo he recibido, creo, pero es necesario que se conozca para que nosotros lo leamos y sepamos y evitemos (S 19).

\section{Discusión}

La búsqueda de información, en la actualidad, no solo se limita a los libros; esto bien lo saben los jóvenes que recurren a la web para diversos fines, entre los que se encuentran descargar material para la elaboración de trabajos académicos. No resulta complicado para un profesor darse cuenta de que un escrito contiene información dudosa. La incoherencia entre párrafos, pérdida de la linealidad semántica del texto, saltos bruscos y errores gramaticales son indicios de que el material no es original, o a veces una redacción demasiado buena en comparación con la forma habitual de escribir del alumno. Pese a que existe una legislación vigente que sanciona el plagio, en los colegios e incluso en las propias universidades parece existir cierta permisividad. Cometer plagio no solo afecta al interés público sino también los derechos morales del autor sobre su obra (Soto, 2012). 
La búsqueda de atajos irregulares se suele justificar entre los jóvenes, quienes ven en los exámenes, trabajos y docentes sus enemigos. No obstante, esta idea equivocada no hace sino fomentar la creencia del éxito académico sustentado en la ley del mínimo esfuerzo. El plagiar o no pasa por una decisión personal. Destacan la motivación intrínseca y la valoración por el estudio, frente a la postura de aquellos que ven el estudio como un medio para un fin. Existe una relación estrecha entre las variables del contexto y la disposición de los estudiantes a plagiar, por ejemplo, evaluar los beneficios y perjuicios del acto, conocer sus implicancias legales y la probabilidad de ser descubierto (Molina, et al., 2011). Diversos autores han centrado su interés en precisar cuáles son las condiciones que llevan a que un estudiante plagie, como son la presión y el apuro en la elaboración de trabajos, la falta de conocimientos sobre los contenidos del curso y el temor a ser desaprobado.

El plagio se ha convertido en una constante en la sociedad, porque no solo se ha instalado en el campo académico, sino que su trascendencia llega a espacios como la música, la literatura, el arte, la política, el cine, la televisión, etc. Conceptos como "robo", "engaño", "fraude" y "copia" están ligados a este proceder. Plagiar implica tomar lo que no es de uno con el fin de utilizarlo en beneficio propio; plagiar también es copiar una idea sin dar el crédito respectivo a su autor. Pese a que su alcance es amplio, son los ámbitos académico y científico los que más han sentido su impacto. El plagio es un fenómeno de gran complejidad no solo por el daño que ocasiona a la propiedad intelectual sino también por la dificultad para combatirlo. La concepción que se tiene de esta acción prohibida no supone un cambio en quien la practica directa o indirectamente, lo que evidencia flexibilidad en los valores. Los entrevistados en el presente estudio asumen el plagio con los términos antes expuestos, lo cual pone en evidencia que son conscientes de la trascendencia del acto. No obstante, como se comenta más adelante, minimizan su impacto dando justificaciones.

Si bien existen comportamientos propios de esta práctica deshonesta (McCabe \& Treviño, 1993; Jordan, 2001; Moeck, 2002; Lambert, Hogan \& Barton, 2003), los entrevistados coinciden en afirmar que copiar información de internet es un acto común y recurrente entre los estudiantes. El ciberplagio, por tanto, es una de las modalidades de plagio que ha ido en aumento en los últimos años. Es por ello, que esto debe recogerse con mayor detalle en las normativas universitarias (Cavanillas, 2008).

La deshonestidad académica es un fenómeno que altera el aprendizaje y el rendimiento de los estudiantes. La realización de prácticas ilícitas con el fin de obtener algún beneficio (buenas notas o reconocimiento) va en aumento, ya que se las considera una forma de obtener beneficios. Quien las realiza no solo es quien copia en un examen o presenta un trabajo ajeno como suyo, sino también quien, siendo testigo de ello, no lo denuncia.

La complicidad es una forma de deshonestidad. Ahora bien, entre los estudiantes esta tolerancia y permisividad son condiciones que les facilitan la aceptación social, entendida como la condición personal de un individuo con respecto a un grupo de referencia (Zavala, Valadez y Vargas, 2008). Existen algunos puntos en los que los entrevistados coinciden, por ejemplo, en que no existen diferencias en el trato hacia aquellos que plagian; y que, hasta cierto punto, el acto de copiar en los exámenes y en los trabajos no representa un problema, prueba de ello es que no se dan tratos diferenciados entre quienes plagian y quienes no. Por otra parte, la complicidad emerge como una actitud común dentro del grupo. Todos apuntan a un objetivo: sacar buenas notas; es por ello que al trabajar en equipo se pasan por alto este y otros actos. La aceptación social es producto de las valoraciones sociales formuladas por los compañeros frente a situaciones o contexto, en este caso la realización de actividades académicas. El liderazgo, la popularidad, el compañerismo, la jovialidad y el respeto están relacionados con dicha aceptación.

Así como el plagio se constituye en un facilitador social, la permisibilidad es un rasgo importante en quien desea integrarse al grupo, es decir, "yo permito que otros plagien porque, al fin de cuentas, no me afecta", o también "yo permito que otros plagien porque en el grupo todos 
plagian". En esto existe una posición clara entre los estudiantes. Por un lado, no justifican este acto, pues lo consideran "malo"; sin embargo, al preguntárseles si "plagiar es normal", los entrevistados aceptaron que es una práctica común e incluso institucionalizada que empieza en el colegio y que llega a convertirse una necesidad en la universidad.

El plagio, además de determinar el grado con el que los estudiantes hacen trampa, permite evidenciar las deficiencias en la adquisición de conocimientos y capacidades. Estas deficiencias afectan a la innovación y al crecimiento económico, objetivos ambos de las instituciones educativas. Un estudiante que hace trampa lo seguirá haciendo una vez que ejerza su profesión, o bien integrará el grupo de profesionales deficientes e incapaces de contribuir con el desarrollo del país. La integridad académica se sustenta en seis valores fundamentales: honestidad, confianza, justicia, respeto, responsabilidad y valor. Que los estudiantes conozcan y asuman estos valores resulta importante como herramienta contra el plagio. Si "no mentir" y "no engañar" son parte de estos valores, el "copiar y pegar" se impone como el comportamiento deshonesto por naturaleza.

En el Perú el delito de plagio, atentatorio contra la propiedad intelectual, se encuentra tipificado en el artículo $219 .^{\circ}$ y otros del Código Penal, modificados y complementados por la Ley 28289. En el presente estudio, al preguntárseles a los estudiantes si el plagio es considerado un delito, las respuestas fueron unánimes: "plagiar no es un delito", al menos no en la universidad, donde este acto es, según esa percepción, normal y necesario. No obstante, existen también posturas que sostienen que el plagio será o no un delito dependiendo de la situación. Para los entrevistados, los productos académicos desarrollados en los cursos no son considerados importantes; y por ello, plagiar durante su desarrollo no es tan grave.

En la cultura estudiantil está arraigada la idea de que "copiar" es un acto normal. No son pocos los casos de alumnos que se sienten orgullosos de sus métodos cada vez más refinados de hacer trampa en los exámenes y de engañar a sus profesores. "Una vez que finaliza la evaluación, el alumno que 'copia' suele no escondérselo a sus compañeros más cercanos; al contrario, lo comparte con su círculo de amigos, con la certeza de que no será reprendido, sino protegido" (Sousa, Conti, Salles y Mussel, 2016, párr. 35). A la pregunta formulada de si plagiar perjudica a quien lo comete, la mayoría de los entrevistados sostuvieron que sí, que dicha acción es un problema que alcanza su máxima expresión en la vida académica. Esta respuesta lleva a suponer que si ocurre una sanción para el plagiador, esta se presentará una vez que la persona esté fuera de la universidad.

Entre los mecanismos para combatir los actos deshonestos, las instituciones académicas implementan una serie de mecanismos como políticas de integridad académica, reglamentos de ética, códigos de conducta, manuales académicos, entre otros. Todos estos mecanismos apuntan a sancionar las infracciones, como es el caso del plagio, cuyas consecuencias pueden ir desde una nota baja o suspensión hasta la pérdida del título o grado obtenidos. No obstante, un acto deshonesto no solo se soluciona imponiendo sanciones y castigos, sino también mediante la prevención. En función de las respuestas dadas por los entrevistados, el plagio es visto como una forma de "atajo" para llegar al éxito académico, además de una demostración de astucia de quien lo comete. A esta habría que sumar la idea que tienen los estudiantes de que plagiar no es malo, siempre y cuando sea justificado, por ejemplo, cuando no se le entiende a un profesor o cuando los cursos son, en su opinión, complejos. Si se tuviera que hacer un balance entre los beneficios y perjuicios de plagiar, los entrevistados se inclinan por lo primero. Ahora bien, al preguntárseles sobre cuáles serían las principales causas del plagio, estos propusieron tres causas puntuales: el desconocimiento de que se está cometiendo plagio, el facilismo y el temor a desaprobar. Estas respuestas evidencian un trasfondo de carácter informativo y conductual. Informativo porque las respuestas llevan a entender que uno plagia porque no sabe que lo está haciendo, es decir, entiende que no se debe copiar ideas ajenas, pero no cómo se aplica esto en el proceso de redacción de trabajos y desarrollo de exámenes. Autores como Sureda et al. (2009) coinciden en afirmar que el comportamiento del profesor, del alumno, el desarrollo de las TIC y los valores sociales 
predominantes, entre otros, constituye causa de plagio, conclusión que coincide con lo dicho por los estudiantes que formaron parte del estudio.

Ante tal hecho, existen posiciones encontradas, pues, por un lado, se encuentran los estudiantes que por considerarlo un acto "normal" creen que no debería ser castigado; y por otro lado, están los que proponen un castigo, aunque este no conlleve expulsión o sanción, sino que la sanción se manifieste en una nueva oportunidad para rendir el examen o presentar el trabajo. Estas respuestas refuerzan la complicidad que existe entre los estudiantes. Asimismo, las universidades tienen un reglamento en el que se precisan las sanciones para los estudiantes que cometan plagio. $\mathrm{Si}$ bien estos documentos son de consulta obligatoria por los estudiantes, todos los entrevistados afirmaron que jamás habían recibido dicho reglamento. Son precisamente estos documentos los que los entrevistados afirman ayudarían a reducir la presencia de este problema.

\section{Referencias}

Batane, T. (2010). Turning to Turnitin to Fight Plagiarism among University Students. Educational Technology \& Society, 13(2), 1-12. Recuperado de: https://www.jstor.org/stable/jeductechsoci.13.2.1

Cavanillas, S. (2008). El ciberplagio en la normativa universitaria. Digithum, 10, 1-6. Recuperado de: https://www.redalyc.org/html/550/55001006/

Castro, Y., Yoplac-Lopez, B., Carpio-Tello, K., Sihuay-Torres, K., \& Cosar-Quiróz, J. (2016). Percepción del plagio académico en estudiantes de odontología. Educación Médica, 19(3), 141-145. Doi: https://doi.org/10.1016/j.edumed.2017.03.003

Cayuela, A., Tauste, A., Seguí, M., Esteve, J., \& Ronda, E. (2015). ¿Cómo medir el plagio entre alumnos universitarios? Revisión de instrumentos utilizados en artículos científicos. En XIII Jornadas de redes de investigación en docencia universitaria. Recuperado de https://rua.ua.es/dspace/bitstream/10045/48824/1/XIII_Jornadas_Redes_15.pdf

Cebrián-Robles, V., Raposo-Rivas, M., Cebrián de la Serna, M., \& Sarmiento-Campos, J. (2018). Percepción sobre el plagio académico de estudiantes universitarios españoles. Educación XXI, 21(2), 105-129. Doi: https://doi.org/10.5944/educxx1.20062

Coulon, A. (1988). La etnometodología. Madrid: Editorial Cátedra.

Díaz, D. (2015). El uso de Turnitin con retroalimentación mejora la probidad académica de estudiantes de bachillerato. Ciencia, Docencia y Tecnología, 51. Recuperado de: http://www.pcient.uner.edu.ar/index.php/cdyt/article/view/66

Egaa. T. (2012). Uso de bibliografía y plagio académico entre los estudiantes universitarios. Revista de Universidad y Sociedad del Conocimiento, 9(2), 18-30. Recuperado de: https://www.learntechlib.org/p/149501/

Ercegovac, Z., \& Richardson, J.V. (2004). Academic dishonesty, plagiarism included, in the digital age: a literature review. College \& Research Libraries, 301-318. Doi: https://doi.org/10.5860/crl.65.4.301

Espinosa, A., Castellarín, M., \& Biagioni, F. (2013). Prácticas Académicas deshonestas en exámenes escritos. Análisis de una muestra de alumnos de $1^{\circ}$ año de la carrera de Psicología de la UNR. V Congreso Internacional de Investigación y Práctica Profesional en Psicología XX Jornadas de Investigación Noveno Encuentro de Investigadores en Psicología del MERCOSUR. Facultad de Psicología, Universidad de Buenos Aires, Buenos Aires

Gibbs, G. (2012). El análisis de datos cualitativos en Investigación Cualitativa. Madrid: Ediciones Morata.

Guba, E. G. (1981). Criterios de credibilidad en la investigación naturalista. En G. Sacristán y A. Gómez (eds.), La Enseñanza: su teoría y su práctica. (pp.148-165). Madrid: Akal.

Hollander, E. (1971). Principios y métodos de psicología social. Buenos Aires: Amorrertu.

Jordan, A. (2001). College student cheating: The role of motivation, perceived norms, attitudes, and knowledge of institutional policy. Ethics \& Behavior, 11(23), 233-247. Doi: https://doi.org/10.1207/S15327019EB1103_3 
Krippendorf, K. (2002). Metodología del análisis de contenido. Teoría y práctica. Barcelona: Paidós.

Kvale, S. (2011). Las entrevistas en investigación cualitativa. Madrid: Ediciones Morata.

Lai, K.W., \& Weeks, J.J. (2009). High school students' understanding of e-plagiarism: some New Zealand observations. CINZS: LTT, 21(1): 1-15. Recuperado de: https://www.otago.ac.nz/cdelt/otago067253.pdf

Lambert, E. G., Hogan, N. L., \& Barton, S. M. (2003). Collegiate academic dishonesty revisited: What have they done, how often have they done it, who does it, and why did they do it? Electronic Journal of Sociology, 7(4), 1-27. Recuperado de: http://www.sociology.org/ejs-archives/vol7.4/lambert_etal.html

Ley 28289. Ley de Lucha contra la Piratería. Congreso de la Republica, 2004.

Ma, H.J., Wan, G. y Lu, E.Y. (2008). Digital cheating and plagiarism in schools. Theory Into Practice, 47,197-203. Doi: https://doi.org/10.1080/00405840802153809

McCabe, D. L., \& Trevino, L. K. (1993). Academic dishonesty: Honor codes and other contextual influences. The Journal of Higher Education, 64(5), 522-538. Doi: https://doi.org/10.1080/00221546.1993.11778446

Moeck, P. G. (2002). Academic dishonesty: Cheating among community college students. Community College Journal of Research and Practice, 26(6), 479-491. Doi: https://doi.org/10.1080/02776770290041846

Molina, F., Velásquez, J. D., Ríos, S., Calfucoy, P. A., \& Cociña, M. (2011). El fenómeno del plagio en documentos digitales: un análisis de la situación actual en el sistema educacional chileno. Revista Ingeniería de Sistemas, 25, 5-28. Recuperado de: http://www.dii.uchile.cl/ ris/RISXXV/plagio.pdf

Pupovac, V., Bilic-Zulle, L. y Petrovecki, M. (2008). On academic plagiarism in Europe. An analytic approach based on four studies. En R. Comas y J. Sureda (coords.). Academic cyberplagiarism. Digithum, $10 . \quad$ Recuperado de http://www.uoc.edu/digithum/10/dt/eng/pupovac_bilic-zulle_petrovecki.pdf

Rebollo-Quintela, N., Espiñeira-Bellón, E., \& Muñoz-Cantero, J. (2017). Atribuciones causales en el plagio académico por parte de los estudiantes universitarios. Revista de Estudios e Investigación en Psicología y Educación, 6, A6-193. Doi: https://doi.org/10.17979/reipe.2017.0.06.2453

Rokeach, M. (1968). Enciclopedia Internacional de Ciencias Sociales (vol. 1). Madrid: Aguilar. Soto, A. (2012). El plagio y su impacto a nivel académico y profesional. E-Ciencias de la Información, 2(1), 1-13. Recuperado de: https://revistas.ucr.ac.cr/index.php/eciencias/article/view/1213

Sousa, R., Conti, V., Salles, A., \& Mussel, I. (2016). Deshonestidad académica: efectos sobre la formación ética de los profesionales de la salud. Revista Bioética, 24(3), 459-468. Doi: https://dx.doi.org/10.1590/1983-80422016243145

Parra, M., \& Briceño, I. (2013). Aspectos éticos en la investigación cualitativa. Enfermería Neurológica, 12(3). 118-121. Recuperado de: http://www.medigraphic.com/cgi$\mathrm{bin} / \mathrm{new} /$ resumen.cgi?IDARTICULO $=46850$

Turnitin. (2018). Índice global de plagio. Recuperado de https://www.turnitin.com/es/papers/indice-global-de-plagio

Sureda, J., Comas, R., Serrano, L., Nava, C., Oliver, M., \& Morey, M. (2009). El plagio y otras formas de deshonestidad académica entre el alumnado de la universidad TecMilenio: resultados generales. Informe de Investigación. Universitat de les Illes Balears. Recuperado de: http://www.ciberplagio.com/universidad/attachment.php?key=51

Vander, J. (1997). Manual de psicología social. Barcelona: Paidós.

Zavala, M., Valadez, M., \& Vargas, M. (2008). Inteligencia emocional y habilidades sociales en adolescentes con alta aceptación social. Revista Electrónica de Investigación Psicoeducativa, 15(2), 319-338. Recuperado de: http://ojs.ual.es/ojs/index.php/EJREP/article/view/1291 